REVISTA MATEMÁTICA COMPLUTENSE

Volumen 12, número 2: 1999

http://dx.doi.org/10.5209/rev_REMA.1999.v12.n2.17114

\title{
The density condition in projective tensor products.
}

\author{
WOLF-DIETER HEINRICHS
}

\begin{abstract}
In this paper we modify a construction due to J. Taskinen to get a Fréchet space $F$ which satisfies the density condition such that the complete injective tensor product $l_{2} \tilde{\theta}_{\epsilon} F_{b}^{\prime}$ does not satisfy the strong dual density condition of Bierstedt and Bonet. In this way a question that remained open in [14] is solved.
\end{abstract}

\section{Introduction and notation}

The density condition (DC) was introduced by S. Heinrich in the context of ultrapowers of locally convex spaces, see [12]. K. D. Bierstedt and $\mathrm{J}$. Bonet investigated the (DC) and the strong dual density condition (SDDC) in [1] - [4]. The (DC) and the (SDDC) play an important role in the theory of Köthe echelon spaces [1] - [4], for extension of linear operators [10], and in the theory of unbounded operator *-algebras [13].

Many locally convex spaces are in fact topological tensor products. In [15], [16], and [7], A. Peris developed a method to define topological properties by operator. By this procedure he obtained good stability properties in injective tensor products. The author studied this method in the case of (DC), (SDDC), and (DF)-spaces in [14]. It was an open problem in [14] to find a Fréchet space $F$ that satisfies (DC) and a $\mathrm{Ba}$ nach space $X$ such that the complete injective tensor product $X \bar{\otimes}_{\varepsilon} F_{b}^{\prime}$ does not satisfy the (SDDC). We will solve that problem by a modification of a counterexample to J. Taskinen, in [17] or [8], 35.9. For this we

Mathematics Subject Classification: 46A04, 46E10.

Servicio Publicaciones Univ. Complutense. Madrid, 1999. 
use duality properties between injective and projective tensor products.

The notation for locally convex spaces is standard. If $E$ is a locally convex space, then $\mathcal{U}(E)$ stands for a basis of absolutely convex closed 0 -neighborhoods and $\mathcal{B}(E)$ stands for the system of all absolutely convex bounded sets in $E$. By $\Gamma(M)$ we denote the absolutely convex hull of $M$. If $E$ and $F$ are locally convex spaces, then $E \otimes_{\epsilon} F$ and $E \otimes_{\pi} F$ stand for the injective tensor product and projective tensor product, respectively. We denote the completions by $E \bar{\otimes}_{\varepsilon} F$ and $E \bar{\otimes}_{\pi} F$, respectively. $L_{b}(E, F)$ means the space of all continuous linear mappings from $E$ into $F$ endowed with the topology of uniform convergence on the bounded sets of $E$. For $K \subset E, L \subset F$, and $M$ is a linear subspace of $L_{b}(E, F)$, then we write $W(K, L):=\{T \in M: T(K) \subset L\}$. If $X$ is a Banach space, then $B_{X}$ denotes its closed unit ball. We denote by $F I N$ the class of all finite-dimensional Banach spaces.

\section{The main result}

We start with some definitions.

Definition 1. (1) Let $F$ denote a metrizable space and $\left(U_{k}\right)_{k=1}^{\infty}$ a countable basis of closed absolutely convex 0 -neighborhoods in $F$. The space $F$ is said to satisfy the density condition -briefly $(D C)$ - if the following holds

$$
\begin{gathered}
\left.\forall\left(\lambda_{k}\right)_{k=1}^{\infty} \subset\right] 0, \infty[\quad \forall n \in N \quad \exists m \in \mathbf{N} \quad \exists M \in \mathcal{B}(F): \\
\bigcap_{k=1}^{m} \lambda_{k} U_{k} \subset U_{n}+M .
\end{gathered}
$$

(2) Let $E$ denote a locally convex space with an increasing fundamental sequence $\left(M_{k}\right)_{k=1}^{\infty}$ of bounded sets. $E$ is said to satisfy the strong dual density condition -briefly (SDDC)- if the following holds

$$
\begin{gathered}
\left.\forall\left(\lambda_{k}\right)_{k=1}^{\infty} \subset\right] 0, \infty[\quad \forall n \in \mathrm{N} \quad \exists m \in \mathrm{N} \quad \exists U \in \mathcal{U}(E): \\
M_{n} \cap U \quad \subset \quad \Gamma\left(\bigcup_{k=1}^{m} \lambda_{k} M_{k}\right)
\end{gathered}
$$

and the space $E$ is said to satisfy the strong dual density condition by operator -briefly (SDDCO)- if the following holds

$$
\left.\forall\left(\lambda_{k}\right)_{k=1}^{\infty} \subset\right] 0, \infty[\forall n \in N \quad \exists m \in N \quad \exists U \in \mathcal{U}(E)
$$




$$
\begin{aligned}
& \exists\left(Q_{k}\right)_{k=1}^{m} \text { lin. op. on } E: \\
& \sum_{k=1}^{m} Q_{k}=I_{E} \text { and } Q_{k}\left(M_{n} \cap U\right) \subset \lambda_{k} M_{k}, \quad k=1, \ldots, m
\end{aligned}
$$

Quasinormable Fréchet spaces and Fréchet-Montel spaces are examples of spaces satisfying (DC), see [12]. By taking polars it follows that the Fréchet space $F$ satisfies (DC) if and only if the strong dual $F_{b}^{\prime}$ satisfies (SDDC), see [1]. It is readily seen, that (SDDCO) implies (SDDC). The strong dual space of an (FBa)-space with (DC) or a (DF)-space satisfying the strict Mackey condition are examples for spaces satisfying (SDDCO), see [14].

The following propositions characterize the (SDDCO) for $F_{b}^{\prime}$, where $F$ is a Fréchet space, by properties in projective and injective tensor products.

Proposition 2. Let $F$ be a Fréchet space and let $X$ be a Banach space. The following assertions are equivalent:

1. $X \bar{\otimes}_{\pi} F\left(X \otimes_{\pi} F\right.$, resp.) satisfies the condition $\left.\forall\left(\lambda_{k}\right)_{k=1}^{\infty} \subset\right] 0, \infty[\quad \forall n \in \mathbf{N} \quad \exists m \in \mathbf{N} \quad \exists M \in \mathcal{B}(F)$ :

$$
\bigcap_{k=1}^{m} \lambda_{k} \bar{\Gamma}\left(B_{X} \otimes U_{k}\right) \subset \bar{\Gamma}\left(B_{X} \otimes U_{n}\right)+\bar{\Gamma}\left(B_{X} \otimes M\right) .
$$

2. $L_{b}\left(X, F_{b}^{\prime}\right)$ satisfies the strong dual density condition (SDDC).

Proof. We are going to prove the result for the complete projective tensor product $X \tilde{\otimes}_{\pi} F$. It is not hard to see that $X \tilde{\otimes}_{\pi} F$ satisfies condition $I$ if and only if $X \otimes_{\pi} F$ does.

The proposition follows by polarity in the pairing $\left\langle X \otimes_{\pi} F, L\left(X, F_{b}^{\prime}\right)\right\rangle$, using the fact $\left(\bar{\Gamma}\left(B_{X} \otimes U_{k}\right)\right)^{\circ}=W\left(B_{X}, U_{k}^{\circ}\right)$. Remark that $W\left(B_{X}, U_{k}^{\circ}\right)$ is $w^{*}$-compact and hence $\Gamma\left(\bigcup_{k=1}^{m} \lambda_{k} W\left(B_{X}, U_{k}^{\circ}\right)\right)$ is closed, see [1], the proof of Proposition 1.2. $\diamond$

Proposition 3. Let $F$ be a Fréchet space. The following assertions are equivalent: 
1. $X \tilde{\otimes}_{\pi} F\left(X \otimes_{\pi} F\right.$, resp.) satisfies the condition

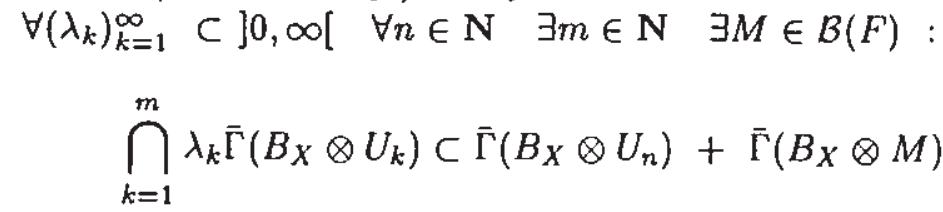

for each Banach space $X$.

2. $F_{b}^{\prime}$ satisfies the strong dual density condition by operator (SD. $D C O)$.

3. $X \otimes_{c} F_{b}^{\prime}\left(L_{b}\left(X, F_{b}^{\prime}\right)\right.$, resp. $)$ satisfies the strong dual density condition (SDDC) for each Banach space $X$.

Proof. By Theorem 1.6 in [14] the strong dual $F_{b}^{\prime}$ satisfies (SDDCO) if and only if $X \dot{\otimes}_{c} F_{b}^{\prime}\left(X \otimes_{\varepsilon} F_{b}^{\prime}\right.$ and $L_{b}\left(X, F^{\prime}\right)$, resp.) has the strong dual density condition (SDDC) for each Banach space $X$. The rest follows by Proposition $2 . \diamond$

Example 4. Let $\lambda$ be an (FM)-sequence space in the class $\Omega$, for the definition of this class see [11]. By the Remark (b) after Proposition 3.2 in [11] the space $X \dot{\otimes}_{\pi} \lambda$ satisfies (DC) for each Banach space $X$. Let $L_{\text {ind }}\left(X, \lambda_{b}^{\prime}\right)$ be the bornological space associated to $L_{b}\left(X, \lambda_{b}^{\prime}\right)$. Then, we omit the proof, $L_{i n d}\left(X, \lambda_{b}^{\prime}\right)$ has (SDDC). By Proposition 3.2 in [11] the topologies on $L_{\text {ind }}\left(X, \lambda_{b}^{\prime}\right)$ and $L_{b}\left(X, \lambda_{b}^{\prime}\right)$ induce the same topology on a bounded subset. Thus $L_{b}\left(X, \lambda_{b}^{\prime}\right)$ has (SDDC) for each Banlach space $X$ and $\lambda_{b}^{\prime}$ satisfies (SDDCO).

We give another argument that $\lambda_{b}^{\prime}$ satisfies (SDDCO). By [5], 3 . Prop. (a) $\lambda$ is a decomposable T-space. It follows by [5], 5. Theorem that $\lambda$ is even an (FBa)-space. We get $L_{b}\left(X, \lambda_{b}^{\prime}\right) \cong\left(X \hat{\Theta}_{\pi} \lambda\right)_{b}^{\prime}$. Since $X \dot{\otimes}_{\pi} \lambda$ has (DC), see [4], 1.7. Corollary, it follows the (SDDC) for $L_{b}\left(X, \lambda_{b}^{\prime}\right)$.

For more examples of Fréchet spaces such that the strong dual spaces satisfy (SDDCO), see [14].

Now we present a slight modification of a counterexample for a (FBa)-space due to J. Taskinen, see [17] or [8], 35.9. We will get the first example for a (DF)-space $E$ such that $E$ satisfies (SDDC) and $E$ does not satisfy (SDDCO). 
We fix a Banach space $X$ that is not an $\mathcal{L}_{1}$-space (for example $X:=$ $l_{2}$ ). Then there are $G_{m, l} \in F I N$ with subspaces $M_{m, l} \subset G_{m, l}$ for $m, l \in$ N such that there exist $z_{m, l} \in X \otimes M_{m, l}$ with

$$
\begin{aligned}
& \pi\left(z_{m, l} ; X, G_{m, l}\right) \leq(m+l)^{-(m+l)^{2}} \text { and } \\
& \pi\left(z_{m, l} ; X, M_{m, l}\right)=(m+l)^{-(m+l)},
\end{aligned}
$$

see [8], 23.5. Let $P_{m, l}: G_{m, l} \longrightarrow G_{m, l}$ be a continuous projection onto $M_{m, l}$ and we define

$$
\|y\|_{m, l, k}:=\left\|P_{m, l}(y)\right\|_{m, l}+k\left\|y-P_{m, l}(y)\right\|_{m, l}
$$

for $m, l, k \in \mathrm{N}$ and $y \in G_{m, l}$. It follows that $\|\cdot\|_{m, l} \leq\|\cdot\|_{m, l, k}$ and $\|y\|_{m, l}=\|y\|_{m, l, k}$ for $y \in M_{m, l}$. Now, we define the spaces:

$$
\begin{array}{cll}
G_{m, l, k}^{\circ}:=\left(G_{m, l},\|\cdot\|_{m, l, k}\right) & ; & G_{m, l, k}:=\left(G_{m, l}\|\cdot\|_{m, l}\right) \\
H^{\circ}:=l_{2}\left(\left(G_{m, l, k}^{\circ}\right)_{m, l, k=1}^{\infty}\right) & ; & H:=l_{2}\left(\left(G_{m, l, k}\right)_{m, l, k=1}^{\infty}\right) .
\end{array}
$$

Then we get $H^{\circ} \subset H$ and $\|\cdot\|_{H} \leq\|\cdot\|_{H^{\circ}}$. If $J_{m, l, k}: G_{m, l, k} \hookrightarrow H$ is the canonical injection, then $z_{m, l, k}:=\left(I_{X} \otimes J_{m, l, k}\right)\left(z_{m, l}\right)$ satisfies

$$
\begin{aligned}
& \pi\left(z_{m, l, k} ; X, H\right) \leq(m+l)^{-(m+l)^{2}} \text { and } \\
& \pi\left(z_{m, l, k} ; X, H^{\circ}\right) \leq(m+l)^{-(m+l)} .
\end{aligned}
$$

We assume that

$$
\begin{aligned}
& \exists m, l, t \in \mathrm{N} \quad \forall k \in \mathrm{N}: \\
& z_{m, l, k} \in \Gamma\left(B_{X} \otimes\left(\frac{1}{2}(m+l)^{-(m+l)} B_{H} \cap t B_{H^{\bullet}}\right)\right) .
\end{aligned}
$$

Then by projection on the $(m, l, k)$-th component of $H$ there is a representation of $z_{m, l}$ by

$$
z_{m, l}=\sum_{i=1}^{N} \lambda_{i} x_{i} \otimes y_{i}=\sum_{i=1}^{N} \lambda_{i} x_{i} \otimes P_{m, l}\left(y_{i}\right) \in X \otimes G_{m, l}
$$

with

$\sum_{i=1}^{N}\left|\lambda_{i}\right| \leq 1, \quad\left\|x_{i}\right\|_{X} \leq 1, \quad\left\|y_{i}\right\|_{m, l} \leq \frac{1}{2}(m+l)^{-(m+l)}, \quad\left\|y_{i}\right\|_{m, l, k} \leq t$. 
Since $P_{m, l}\left(y_{i}\right) \in M_{m, l}$, it follows that

$$
\begin{aligned}
(m+l)^{-(m+l)} & =\pi\left(z_{m, l} ; X, M_{m, l}\right) \leq \sum_{i=1}^{N}\left|\lambda_{i}\right|\left\|x_{i}\right\|_{X}\left\|P_{m, l}\left(y_{i}\right)\right\|_{m, l} \\
& \leq \sum_{i=1}^{N}\left|\lambda_{i}\right|\left(\left\|y_{i}\right\|_{m, l}+\left\|y_{i}-P_{m, l}\left(y_{i}\right)\right\|_{m, l}\right) \\
& \leq \frac{1}{2}(m+l)^{-(m+l)}+\frac{t}{k}
\end{aligned}
$$

This is a contradiction for large $k$ and it follows that

$$
\begin{aligned}
& \forall m, l, t \in \mathrm{N} \quad \exists k \in \mathrm{N}: \\
& z_{m, l, k} \notin \Gamma\left(B_{X} \otimes\left(\frac{1}{2}(m+l)^{-(m+l)} B_{H} \cap t B_{H^{\circ}}\right)\right) .
\end{aligned}
$$

Now set for brevity

$$
f_{n, m, l}(x):=(n+1)^{(m+l)}\|x\|_{H^{\circ}} \vee(n+1)^{(m+1)^{2}}\|x\|_{H} \text { for } x \in H^{\circ}
$$

and

$$
g_{n, m, l}(x):=(n+1)^{(m+l)^{2}}\|x\|_{H} \quad \text { for } \quad x \in H .
$$

Then the Fréchet space $F_{0}$ is defined as double sequence space

$$
\begin{array}{r}
\left\{\left(x_{i j}\right)_{i, j=1}^{\infty} \subset H^{\circ}:\left(\left(f_{n, m, l}\left(x_{i j}\right)\right)_{i=1, \ldots, \infty ; j<n},\left(g_{n, m, l}\left(x_{i j}\right)\right)_{i=1, \ldots, \infty ; j \geq n}\right)\right. \\
\left.\in l_{\infty}(\mathbf{N} \times \mathbf{N}) \quad \forall n \in \mathbf{N}\right\},
\end{array}
$$

and a decreasing basis of 0 -neighborhoods is given by $\left(U_{n}\right)_{n=1}^{\infty}$, where

$$
\begin{aligned}
U_{n}:= & \left(\left(C_{n, i, 1} \cap D_{n, i, 1}\right)_{i}, \ldots,\left(C_{n, i, n-1} \cap D_{n, i, n-1}\right)_{i}\right. \\
& \left.\left(D_{n, i, n}\right)_{i}, \ldots,\left(D_{n, i, j}\right)_{i}, \ldots\right) \\
& \uparrow_{n} \uparrow_{j} \\
C_{n, i, j}:= & \frac{B_{H^{\circ}}}{(n+1)^{(i+j)}}, \quad D_{n, i, j}:=\frac{B_{H}}{(n+1)^{(i+j)^{2}}}
\end{aligned}
$$

and $(. .)_{i}$ stands for a column vector. 
Lemma 5. The Fréchet space $F_{0}$ satisfies the density condition (DC).

Proof. Given any positive sequence $\left(\lambda_{k}\right)_{k=1}^{\infty}$ and $n \in \mathrm{N}$. Choose $m>n$ with $\frac{\lambda_{n+1}}{(n+2)^{m}} \leq \frac{1}{(n+1)^{m}}$. This implies $\frac{\lambda_{n+1}}{(n+2)^{(i+2)}} \leq \frac{1}{(n+1)^{(i+2)}}$ and $\frac{\lambda_{n+1}}{(n+2)^{(i+j)^{2}}} \leq \frac{1}{(n+1)^{(i+j)^{2}}}$ for all $i+j \geq m$ and the following relations

$$
\begin{aligned}
\lambda_{n+1} C_{n+1, i, j} & =\frac{\lambda_{n+1}}{(n+2)^{(i+j)}} B_{H^{\circ}} \subset \frac{1}{(n+1)^{(i+j)}} B_{H^{\circ}}=C_{n, i, j} \text { and } \\
\lambda_{n+1} D_{n+1, i, j} & \subset D_{n, i, j}
\end{aligned}
$$

for $i+j \geq m$. By a short calculation we get

$$
\begin{aligned}
& \lambda_{n+1} U_{n+1} \cap \lambda_{m} U_{m} \subset \\
& \left(\begin{array}{cccccc}
\lambda_{m} C_{m, 1,1}, & \lambda_{m} C_{m, 1,2}, & \ldots, & \lambda_{m} C_{m, 1, m-1}, & 0, & \ldots \\
\lambda_{m} C_{m, 2,1}, & \lambda_{m} C_{m, 2,2}, & \ldots, & \lambda_{m} C_{m, 2, m-1}, & 0, & \ldots \\
\ldots, & \ldots, & \ldots, & \ldots, & \ldots, & \ldots \\
\lambda_{m} C_{m, m-1,1}, & \lambda_{m} C_{m, m-1,2}, & \ldots, & \lambda_{m} C_{m, m-1, m-1}, & 0, & \ldots \\
0, & 0, & \ldots, & 0, & 0, & \ldots \\
\ldots, & \ldots, & \ldots, & \ldots, & \ldots, & \ldots
\end{array}\right) \\
& +\left(\begin{array}{ccccc}
0, & \ldots, & 0, & D_{n, 1, m}, & \ldots \\
\ldots, & \ldots, & \ldots, & \ldots, & \ldots \\
0, & \ldots, & 0, & D_{n, m-1, m}, & \ldots \\
C_{n, m, 1} \cap D_{n, m, 1}, & \ldots, & D_{n, m, m-1}, & D_{n, m, m}, & \ldots \\
C_{n, m+1,1} \cap D_{n, m+1,1}, & \ldots, & D_{n, m+1, m-1}, & D_{n, m+1, m}, & \ldots \\
\ldots, & \ldots, & \ldots, & \ldots, & \ldots
\end{array}\right) \\
& \text { C } M+U_{n} \text {, }
\end{aligned}
$$

where $M$ is a bounded subset of $F_{0}$. Hence

$$
\bigcap_{k=1}^{m} \lambda_{k} U_{k} \subset \lambda_{n+1} U_{n+1} \cap \lambda_{m} U_{m} \subset M+U_{n}
$$

and the lemma is proved. $\diamond$ 
Theorem 6. Let $X$ be a Banach space that is not an $\mathcal{L}_{1}$-space and let $F_{0}$ be constructed as above. Then $\left(F_{0}\right)_{b}^{\prime}$ satisfies the strong dual density condition $(S D D C)$ and $L_{b}\left(X,\left(F_{0}\right)_{b}^{\prime}\right)$ does not satisfy the (SDDC). The space $\left(F_{0}\right)_{b}^{\prime}$ does not satisfy the strong dual density condition by operator (SDDCO).

Proof. Denote by $J_{i, j}$ the canonical injection of the $(i, j)$-th component into $F_{0}$ and by $Q_{i, j}$ the corresponding projection. Further let $q_{n}(\cdot)$ denote the $n$-th seminorm in $X \Theta_{\pi} F_{0}$. By (2), (4) and (4) we get

$$
\begin{aligned}
& q_{n}\left(\left(I_{X} \odot J_{l, m}\right)\left(z_{m, l, k}\right)\right) \leq \frac{(n+1)^{(m+l)^{2}}}{(m+1)^{(m+l)^{2}}} \vee \frac{(n+1)^{(m+l)}}{(m+1)^{(m+l)}}<\varrho_{n} \\
& \text { for } m<n \text { and } \\
& q_{n}\left(\left(I_{X} \otimes J_{l, m}\right)\left(z_{m, l, k}\right)\right) \leq \frac{(n+1)^{(m+l)^{2}}}{(m+l)^{(m+l)^{2}}} \leq 1 \text { for } m \geq n .
\end{aligned}
$$

It follows that the set $A:=\left\{\left(I_{X} \otimes J_{l, m}\right)\left(z_{m, l, k}\right): m, l, k \in \mathrm{N}\right\}$ is bounded. There exists positive $\left(\lambda_{j}\right)_{j=1}^{\infty}$ such that $A \subset \bigcap_{j=1}^{\infty} \lambda_{j} \Gamma\left(B_{X^{\circ}} \bigcirc\right.$ $U_{j}$ ). We assume $X \vartheta_{\pi} F_{0}$ satisfies the condition 1 in Proposition 2. Following for each $n \in \mathrm{N}$ exist $p_{n} \in \mathrm{N}$ and $M_{n} \subset F_{0}$ bounded such that

$$
\begin{aligned}
A \subset \bigcap_{j=1}^{p_{n}} \lambda_{j} \Gamma\left(B_{X} \ominus U_{j}\right) & \subset \Gamma\left(B_{X} \odot U_{n+1}\right)+\bar{\Gamma}\left(B_{X} \odot M_{n}\right) \\
& \subset 2 \Gamma\left(B_{X} \odot U_{n+1}\right)+\Gamma\left(B_{X} \odot M_{n}\right) .
\end{aligned}
$$

There are $\rho_{i}^{n} \geq 1$ with $M_{n} \subset \bigcap_{i=1}^{\infty} \rho_{i}^{n} U_{i}$ and we get

$$
A \subset 3 \Gamma\left(B_{X} \odot\left(\rho_{1}^{n} U_{1} \cap \rho_{n+1}^{n} U_{n+1}\right)\right) \text {. }
$$

Thus

$$
\begin{aligned}
z_{n, l, k} & =\left(I_{X} \odot Q_{l, n}\right)\left(I_{X} \odot J_{l, n}\right)\left(z_{n, l, k}\right) \\
& \in\left(I_{X} \ominus Q_{l, n}\right) \Gamma\left(B_{X} \odot\left(3 \rho_{1}^{n} U_{1} \cap 3 \rho_{n+1}^{n}\left(j_{n+1}\right)\right)\right. \\
& \subset \Gamma\left(B_{X} \odot\left(\frac{3 \rho_{1}^{n}}{2^{(n+l)^{2}}} B_{H} \cap \frac{3 \rho_{n+1}^{n}}{(n+2)^{(n+l)}} B_{H}\right)\right)
\end{aligned}
$$


Now, for arbitrary $n \in \mathrm{N}$ choose $l \in \mathrm{N}$ with $\frac{3 \rho_{1}^{n}}{2^{(n+1)^{2}}} \leq \frac{1}{2(n+1)(n+l)}$ and set $t:=\frac{3 \rho_{n+1}^{n}}{(n+2)^{(n+1)}}$. Thus

$$
z_{n, l, k} \in \Gamma\left(B_{X} \otimes\left(\frac{1}{2(n+l)^{(n+l)}} B_{H} \cap t B_{H^{\circ}}\right)\right)
$$

for all $k \in \mathrm{N}$ that is a contradiction to equation (3). It follows that $X \vartheta_{\pi} F_{0}$ does not satisfy condition $I$ in Proposition 2 and $L_{b}\left(X_{+}\left(F_{0}\right)_{b}^{\prime}\right)$ does not satisfy (SDDC). Clearly, the space $\left(F_{0}\right)_{b}^{\prime}$ has (SDDC) by Lemma 5 and does not satisfy (SDDCO) by Proposition 3. $\diamond$

Corollary 7. If $F_{0}$ is the Fréchet space constructed above, the space $L_{b}\left(I_{2},\left(F_{0}\right)_{b}^{\prime}\right)$ does not satisfy the strong dual density condition (SDDC).

\section{References}

[1] Bierstedt, K. D. and Bonet, J., Stefan Heinrich's Density Condition for Fréchet Spaces and the Characterization of the Distinguished Köthe Echelon Spaces, Math. Nachr. 135 (1988), 149-180.

[2] Bierstedt, K. D. and Bonet. J., Dual density conditions in (DF)spaces I, Results in Math. 14 (1988), 242-274.

[3] Bierstedt, K. D. and Bonet. J.. Dual density conditions in (DF)spaces II, Bull. Soc. Roy. Sci. Liège 57 (1988), 567-589.

[4] Bierstedt. K. D. and Bonet, J.. Density Condition in Fréchet and (DF)-spaces, Rev. Mat. Liniv. Compl. Madrid 2 (1989), 59-76.

[5] Bonet, J. and Díaz, J. (... The problem of topologies of Grothendieck and the class of Fréchet T-spaces, Math. Nachr. 150 (1991), 109-118.

[6] Bonet, J., Díaz, J. C. and Taskinen, J., Tensor stable Fréchet and (DF)-spaces. Collect. Math. 42.3 (1991), 199-236.

[7] Boyd, C. and Peris, A., A projective description of the Nachbinported topology, J. Math. Anal. Appl. 197, 3 (1996), 635-657. 
[8] Defant, A. and Floret, K., Tensor Norms and Operator Ideals, North-Holland Math. Studies 176, North Holland, Amsterdam, 1993.

[9] Defant, A., Floret, K. and Taskinen J., On the injective tensor product of (DF)-spaces, Archiv Math. 57 (1991), 149-154.

[10] Defant, A, and Peris, A., Maurey's extensions theorem and Grothendieck's problème des topologies, J. London Math. Soc. , to appear.

[11] Diaz, J. C. and Molina, J.A.L., On the Projective Tensor Product of Fréchet Spaces, Proc. Edinburgh Mat. Soc. 34 (1991), 169-178.

[12] Heinrich, S., Ultrapowers of Locally Convex Spaces and Applications, Math. Nachr. 118 (1984), 285-315.

[13] Heinrichs, W.-D., The Density Property in Fréchet-Domains of Unbounded Operator *-algebras, Math. Nachr. 165 (1994),49-60.

[14] Heinrichs, W.-D., The Density Condition and the Strong Dual Density Condition by Operator, Collect. Math. 48, 3 (1997), 321-337.

[15] Peris, A., Quasinormable Spaces and the Problem of Topologies of Grothendieck, Ann. Acad. Sci. Fenn. Ser. A. 19 (1994), 167-203.

[16] Peris, A. and Rivera M. J., Localisation of Bounded Sets in Tensor Products, Rev. Mat. Univ. Compl. Madrid 9 (1996), 111-130.

[17] Taskinen, J., Counterexamples to "problème des topologies" of Grothendieck, Ann. Acad. Sci. Fenn. Ser. A. 63 (1986).

Wolf-Dieter Hein richs

Fakultät für Mathematik und Informatik

Universität Leipzig

Augustusplatz

D-04109 Leipzig

Germany

Recibido: 17 de Noviembre de 1997

Revisado: 8 de Febrero de 1999 